\title{
Stereotactic body radiotherapy for prostate cancer: ready for prime time?
}

\begin{abstract}
Alan Katz
Received: 25 January 2012 / Accepted: 30 January 2012 /Published online: 14 February 2012

(C) Springer-Verlag 2012
\end{abstract}

\begin{abstract}
Stereotactic body radiotherapy (SBRT) delivers a few high doses of radiation to a target in the body with extreme accuracy and conformality. Prostate cancer's great sensitivity to dose per fraction allows SBRT's hypofractionated delivery the opportunity to increase the biological equivalent dose delivered to the prostate while relatively decreasing the dose to the normal bladder and rectal tissues. In recent years, clinical outcomes supporting the safety and increasingly longer-term efficacy of SBRT have been published. This review examines the basis of hypofractionation, current SBRT delivery mechanisms, and the growing body of clinical outcomes of SBRT for prostate cancer. In addition, given the increased interest in SBRT for prostate cancer, clinical issues in the treatment of prostate cancer with SBRT and a look into the future of SBRT for prostate cancer are examined.
\end{abstract}

Keywords Prostate Stereotactic body radiotherapy CyberKnife

\section{Introduction}

Stereotactic body radiotherapy (SBRT), delivery of a few high doses of radiation to a target in the body with extreme accuracy and conformality appears well suited for treatment of localized prostate cancer. Foremost in this supposition is the increase in therapeutic ratio offered by SBRT. Specifically, prostate cancer has great sensitivity to dose per

\section{A. Katz $(\triangle)$}

Flushing Radiation Oncology,

40-20 Main St.,

Flushing, NY 11354, USA

e-mail: akatzmd@msn.com fraction ( $\alpha / \beta$ ratio of $\sim 1.5 \mathrm{~Gy}$ ) [1-3], whereas the $\alpha / \beta$ ratio for late damage to the bladder and rectum is 3-5 Gy [4]. This provides SBRT's hypofractionated delivery an opportunity to increase the biological equivalent dose delivered to the prostate while relatively decreasing the dose to the normal bladder and rectal tissues. While the fundamental concept of SBRT is relatively straightforward, debate focused on prostate cancer's $\alpha / \beta$ ratio initially hindered application of SBRT for localized prostate cancer $[5,6]$. Nevertheless, usage of SBRT for localized prostate cancer has steadily increased in recent years as clinical outcomes supporting the safety and increasingly longer-term efficacy of SBRT are published [7-13]. Indeed the interest in SBRT for localized prostate cancer has recently resulted in the radiation therapy oncology group (RTOG) opening a randomized non-inferiority phase II trial, RTOG 0938, comparing delivery of $36.25 \mathrm{~Gy}$ in five fractions over 2 weeks to 51.6 Gy delivered in 12 fractions over 2.5 weeks [14]. This review examines the basis of hypofractionation, current SBRT delivery mechanisms, and clinical outcomes of SBRT for prostate cancer. In addition, given the increased interest in SBRT for prostate cancer, clinical issues in the treatment of prostate cancer with SBRT and a look into the future of SBRT for prostate cancer are examined.

\section{The case for hypofractionation}

Conventional fractionation for prostate cancer delivers 3845 fractions of 1.8-2.0 Gy over a course of 7-9 weeks whereas SBRT delivers four to six fractions of 35-50 Gy over a course of 1-2 weeks. Given the large difference in the number of fractions and dose per fraction, clinical acceptance of SBRT has been a long and very gradual process involving incremental changes in the number of fractions 
delivered. Most hypofractionated dose schemes over the past 10-15 years employed so called "moderate hypofractionation" that delivers $20-28$ fractions of 2.5-3.0 Gy. For example, Kupelian et al. [15] delivered 70 Gy in 28 fractions using intensity-modulated radiation therapy (IMRT) to 770 patients. The prescription dose was delivered to the prostate with $8 \mathrm{~mm}$ margins that were reduced to $4 \mathrm{~mm}$ posteriorly. At a median follow-up of 45 months, the 5-year actuarial freedom from biochemical failure rate was $95 \%$ for low-risk and $85 \%$ for intermediate-risk patients, using the Phoenix (nadir +2 ) definition of biochemical failure. Toxicity was acceptably low. Researchers at McGill University [16] delivered 66 Gy in 22 fractions to 129 patients using threedimensional (3D) conformal external beam radiation therapy (EBRT). At a median 51 months follow-up, the 5-year actuarial freedom from biochemical failure rate was $98 \%$ for both the low- and intermediate-risk patients. Rectal toxicity was somewhat higher than in the Kupelian et al. study, one possible cause of which may have been the higher rectal dose with 3D conformal delivery compared to that with IMRT delivery.

Recently, randomized trials comparing moderate hypofractionation to conventional fractionation have emerged with 3-7 year results. Arcangeli et al. [17] reported a trial of 168 high-risk patients who were randomized to receive either 80 Gy in 2 Gy fractions or 62 Gy in 3.1 Gy fractions. Both arms were delivered with 3D conformal EBRT. At a median follow-up of 32 and 35 months in the moderate hypofractionation and conventional fractionation arms, respectively, there was a clear superiority in biochemical control for the hypofractionated arm with a 3-year actuarial freedom from biochemical failure rate of $87 \%$ for the moderate hypofractionation and $79 \%$ for the conventional fractionation arm. Toxicity was not statistically different between the two arms. Most recently, at ASTRO 2011, Pollack et al. [18] reported on a randomized trial comparing $76 \mathrm{~Gy}$ delivered in 38 fractions to 70.2 Gy delivered in 26 fractions using IMRT. A total of 303 intermediate- and high-risk patients with a median follow-up of more than 60 months for the surviving patients were assessed. At five years, no statistically significant difference in late toxicity or freedom from biochemical failure was observed. Decreasing fraction number to an even greater extent, Ritter et al. [19] reported at ASTRO 2011 on three increasing dose per fraction schedules (64.7 Gy in 22 fractions, 58.08 Gy in 16 fractions, and 51.6 Gy in 12 fractions) all delivered with IMRT. A total of 317 low- and intermediate-risk patients had a median followup of 56, 37, and 28 months per fraction schedule. The 5-year freedom from biochemical failure rate was $91.5 \%$ for the 64.7 Gy dose, and the 3-year freedom from biochemical failure rates were $96.1 \%$ and $98.7 \%$ for the other two arms.

Combined, the toxicity and efficacy results for these studies of moderate hypofractionation [16-18, 20] support the concept of a low $\alpha / \beta$ ratio and the use of increased hypofractionation (i.e., decreased fraction number with increased dose per fraction). Indeed, in comparison to the above studies, Zelefsky et al. [21]delivered conventional fractionation of 81 Gy in 45 fractions with IMRT to 561 patients. The actuarial 5-year freedom from biochemical failure rates were $89 \%, 78 \%$, and $72 \%$ for the favorable-, intermediate-, and unfavorable-risk patients, respectively. As can be seen in Table 1, these results of hypofractionated treatment described above, which is consistent with an $\alpha / \beta$

Table 1 Overview of conventional fractionation, moderate hypofractionation and HDR brachytherapy hypofractionation results

\begin{tabular}{|c|c|c|c|c|c|}
\hline Study & Treatment & Risk & EQD1.8 & Grade $3+$ late toxicity & FFBF \\
\hline \multicolumn{6}{|c|}{ Conventional fractionation } \\
\hline Zelefsky [21] & $81 \mathrm{~Gy}$ in $45 \mathrm{fx}$ & Low, int, and high & 81 Gy & $3 \%$ GU, $0.1 \%$ GI & $\begin{array}{l}89 \%, 78 \% \text {, and } \\
72 \% 8 \text {-year by risk }\end{array}$ \\
\hline Arcangeli [17] & $80 \mathrm{~Gy}$ in $40 \mathrm{fx}$ & High & 83 Gy & NSBD & $79 \%$ 3-year \\
\hline Pollack [18] & $76 \mathrm{~Gy}$ in $38 \mathrm{fx}$ & NS & 84 Gy & NS & $79 \% 5$-year \\
\hline \multicolumn{6}{|c|}{ Increasingly hypofractionated } \\
\hline Kupelian et al. [15] & $70 \mathrm{~Gy}$ in $28 \mathrm{fx}$ & Low, int, and high & 84 Gy & $0.1 \% \mathrm{GU}, 1.4 \% \mathrm{GI}$ & $\begin{array}{l}94 \%, 83 \% \text {, and } \\
72 \% 5 \text {-year by risk }\end{array}$ \\
\hline Rene et al. [16] & 66 Gy in $22 \mathrm{fx}$ & Low & 90 Gy & $\begin{array}{l}4 \% \text { Late grade } 3 \mathrm{GU}, \\
25 \% \text { late grade } 2 / 3 \mathrm{GI}\end{array}$ & $98 \% 5$-year \\
\hline Arcangeli et al. [17] & $62 \mathrm{~Gy}$ in $20 \mathrm{fx}$ & High & 87 Gy & NSBD & $87 \%$ 3-year \\
\hline Pollack et al. [18] & 70.2 Gy in $26 \mathrm{fx}$ & NS & 84 Gy & NS & $79 \% 5$-year \\
\hline Ritter et al. [19] & $\begin{array}{l}64.7 \mathrm{~Gy} \text { in } 22 \mathrm{fx} \\
58.08 \text { in } 16 \mathrm{fx} \\
51.6 \mathrm{~Gy} \text { in } 12 \mathrm{fx}\end{array}$ & Low and int & 87.9 Gy & NSBD & $\begin{array}{l}91.5 \% \text { 5-year } \\
96.1 \% \text { 3-year } \\
98.7 \% \text { 3-year }\end{array}$ \\
\hline
\end{tabular}

The comparable freedom from biochemical failure (FFBF) rates and moderate toxicity of increasingly hypofractionated results support a low $\alpha / \beta$ ratio and the use of even higher doses per fraction

$f x$ Fractions, int intermediate, EQD equivalent dose at 1.8 Gy per fraction, assuming an $\alpha / \beta$ of $1.5 \mathrm{~Gy}$, NS not specified, $N S B D$ not specified by dose 
ratio of $1.5 \mathrm{~Gy}$. That is, if the $\alpha / \beta$ ratio were significantly higher than $1.5 \mathrm{~Gy}$, then hypofractionated studies [16-18, 20] would have EQD1.8's below $70 \mathrm{~Gy}$, which contradicts their comparable outcomes to conventional fractionation delivery at $81 \mathrm{~Gy}$.

\section{Increased hypofractionation: SBRT}

Further increasing the dose-per-fraction is sometimes referred to as extreme hypofractionation. The first use of hypofractionation for prostate cancer occurred in England during the 2D planning era from 1965 to 1980 [22]. It appears economic factors motivated the adaptation of a 36 Gy total dose delivered in 6 fractions every other day (QOD), as England had a capacity shortage and was unable to treat all of its patients with more prolonged schedules. Although prostate-specific antigen (PSA) data did not exist at that time, the patients had a good clinical response with overall survival curves equivalent to the population at large. Toxicity was surprisingly low considering the large irradiation fields and lack of image guidance, probably because the hypofractionated scheme drove down the equivalent dose to normal tissues. Nevertheless, for many subsequent years, hypofractionation with EBRT was abandoned.

Around 1990, hypofractionation was resurrected using internal applications of high-dose rate (HDR) brachytherapy. This precise and conformal technique for radiation delivery was initially used as a boost to conventional EBRT, followed more recently by HDR brachytherapy monotherapy. Both approaches have yielded excellent tumor control with reasonably low morbidity [23-25]. For instance, Demanes et al. used HDR brachytherapy as a boost to 45 Gy EBRT. The 10 -year freedom from biochemical failure rates were $87 \%$ and $69 \%$ for intermediate- and high- risk patients, respectively. Martinez et al. [24] reported 5-year freedom from biochemical failure rates of $91 \%$ and $88 \%$ in a total of 248 low-risk patients treated using a total dose of 38 Gy delivered in four fractions or 42 Gy delivered in six fractions, respectively. Demanes et al. reported a 5- and 8-year freedom from biochemical failure rate of $97 \%$ for 298 low- and low-intermediate-risk patients treated with a total dose of 38 Gy delivered in four fractions or $42 \mathrm{~Gy}$ delivered in six fractions. Most recently, Yoshioka et al. [26] report 5-year freedom from biochemical failure rates of $85 \%, 93 \%$, and $79 \%$ for low-, intermediate-, and high-risk patients treated with a total dose of 54 Gy delivered in nine fractions. These studies also reported low toxicity, demonstrating both the safety and efficacy of HDR brachytherapy monotherapy. However, HDR brachytherapy delivery requires hospitalization, catheterization and is uncomfortable for the patient particularly for multiday delivery regimens where by the delivery needles remain inserted into the patient for an extended time period. Consequently, the question arose as to whether the success of HDR brachytherapy could be emulated in an alternative hypofractionated approach that was also a noninvasive, outpatient treatment [27]. Specifically, if four to five fractions of SBRT could take advantage of the low $\alpha / \beta$ ratio in a noninvasive, cost-saving way, it was thought to carry an advantage over HDR brachytherapy and more conventional dose schedules with EBRT.

\section{SBRT delivery}

The advent of image-guided delivery technologies in the early 2000s with improved accuracy opened the doors for SBRT delivery which requires highly accurate delivery due to the high dose delivered per fraction. Using conventional LINAC techniques without real-time target imaging, several millimeters of systematic and random error are known to occur due to inter- and intrafraction movement of the prostate relative to the bony anatomy [28]. However, these inaccuracies can be overcome by modern LINAC technologies with image guidance. Today, gantry-based linear accelerators (LINACs), such as Trilogy, Novalis Tx, and TrueBeam, by Varian Medical Systems (Palo Alto, CA); and Synergy and Axesse by Elekta (Stockholm) provide on-board cone beam computed tomography (CT) imaging provide accuracy in daily set-up. TomoTherapy by Accuray Inc. (Sunnyvale, CA) is a ring gantry that uses fan-beam megavoltage CT scans for accurate daily set-up. Alone, however, these systems do not provide intrafraction guidance which can be significant. For instance, Kron et al. [29] examined intrafraction motion for 184 patients using implanted fiducials and found a mean motion of $1.7 \mathrm{~mm}$ (range, 0 to $25 \mathrm{~mm}$ ) over 3-30 min time intervals. Several approaches exist for intrafraction motion guidance. The ExacTrac X-ray 6D system by Brain Lab with a robotic couch provides real-time image guidance when integrated with Varian Medical Systems LINACs. Calypso's (Calypso Medical Systems, Seattle, WA) electromagnetic transponders allow continuous detection of prostate translations which, when coupled with manual intervention, permit correction of patient positioning. The CyberKnife by Accuray Inc. is a $6 \mathrm{MV}$ LINAC placed at the end of a three-jointed, 6 degrees-of-freedom robotic arm and a robotic treatment couch with $6^{\circ}$ of freedom. CyberKnife SBRT employs fiducial markers placed in the prostate to verify organ position in real time via a pair of orthogonal electronic X-ray imaging devices. The positional information is used to perform real time corrections for position during the treatment by overlaying the images obtained on digitally reconstructed radiographs from the planning CT scan.

The first study to examine the feasibility of SBRT for prostate cancer [30] focused on the CyberKnife [30]. This treatment planning study demonstrated the ability of the CyberKnife to deliver highly conformal plans suitable for 
SBRT like doses. As King et al. pointed out, the CyberKnife offers excellent dosimetric and motion tracking features, ideal for SBRT, especially for a moving target like the prostate. They particularly emphasized the advantage of intrafractional tracking and automatic correction of beam aim to decrease uncertainty in prostate position, thus allowing reduction of the amount of normal tissue included in the planning volume. The conformality of treatment plans produced with CyberKnife have been shown to be superior to IMRT by Hossain et al. [31] who performed treatment planning for eight patients with both the Accuray Multiplan system and a nine-field IMRT system. The CyberKnife plans had better conformality indices (1.18 vs. 1.44$)$ than the IMRT plans, with slightly higher dose inhomogeneity in the target. The dose fall-off was similar in both plans. This was corroborated by Dawood et al. [32], who showed that the ability to decrease the PTV expansion from 8 to $5 \mathrm{~mm}$ with the CyberKnife resulted in better dose volume histograms (DVHs) to the bladder and rectum. Hossain et al. [31] also studied the intrafraction motion of the prostate during a 50-70 min treatment and its potential dosimetric effect. The effect of movement was case-dependent. Although most cases did not display enough movement to affect the V100 substantially, sporadic motion was seen which could reduce the V100 by $10 \%$. The authors conclude that motion management is essential in providing optimal hypofractionated doses to the prostate. Xie et al. [33] examined intrafraction motion in 21 patients treated with the CyberKnife at Stanford University. They concluded that that reimaging every $40 \mathrm{~s}$ is sufficient in most cases to ensure submillimeter accuracy. They also found no evidence of prostate deformation during treatment. Lei et al. [34] recently examined their motion tracking experience with the CyberKnife at Georgetown University. Using three to four fiducials, they were able to perform full six-dimensional (6D) tracking (translational, rotational, and pitch) in 86 of 88 cases. Since the prostate may pitch anteriorly from rectal gas, they emphasize the importance of $6 \mathrm{D}$ tracking, which is unique to the CyberKnife. All of these studies emphasize the need to manage motion in order to keep the high dose of radiation delivered with SBRT to a minimal margin of tissue around the prostate.

\section{SBRT dosimetry}

SBRT for prostate cancer uses one of two general dosimetric approaches: homogeneous or heterogeneous. The most common approach is the homogeneous dose distribution which is possible with both the CyberKnife (Fig. 1) and gantry based LINAC delivery (Fig. 2). For the CyberKnife, the homogeneous dose entails treating the PTV to a minimum of $83-87 \%$ of the maximum dose. For the gantry-based LINACS, such as Trilogy, Novalis, and TomoTherapy,
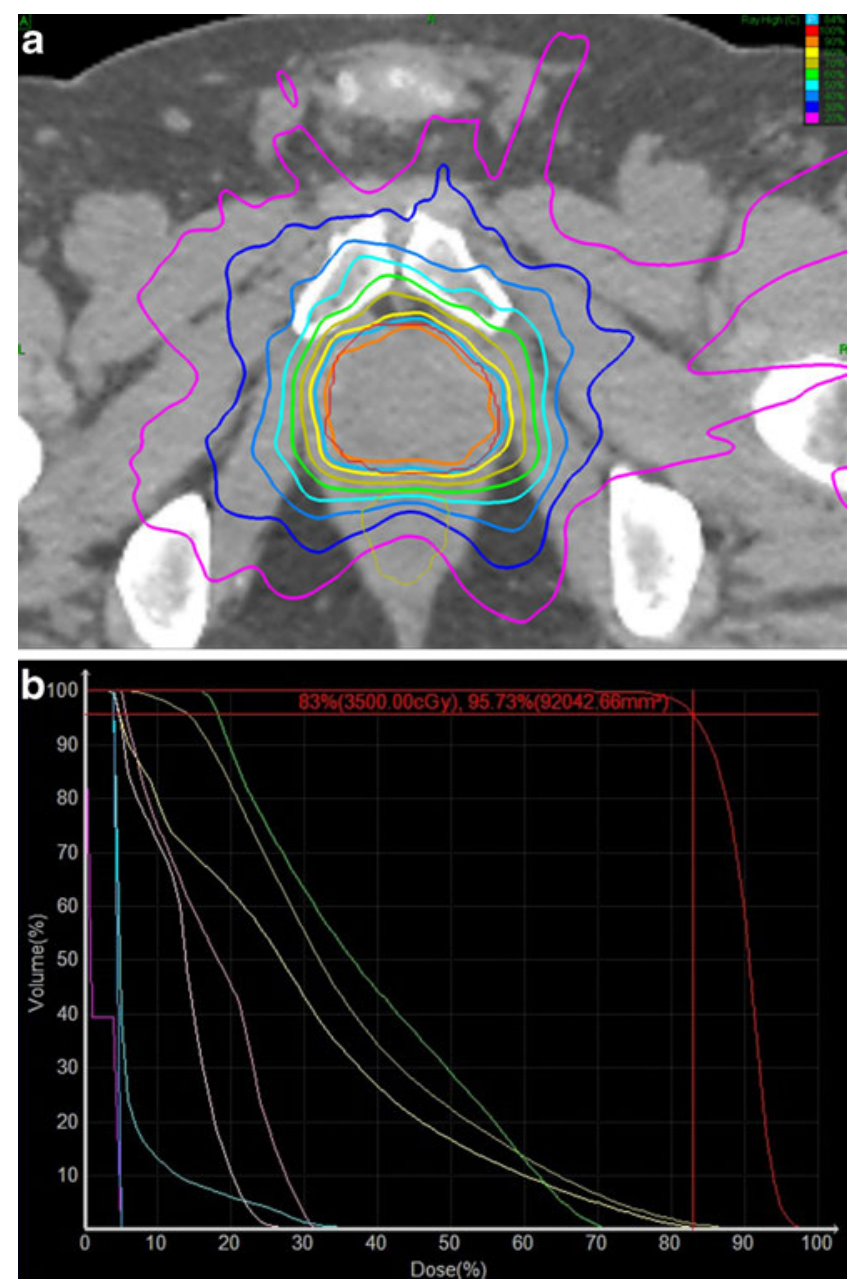

Fig. 1 Example homogeneous CyberKnife treatment plan with a dose of 35 Gy delivered in five fractions to the $84 \%$ line. a Treatment plane with PTV in red. b Dose volume histogram. Shown from left to right are the testes, left femoral head, right femoral head, bladder, rectum, penile bulb and PTV. Image courtesy Jinghao Zhou, PhD Flushing Radiation Oncology, Flushing NY

typically the PTV covers by $90 \%$ using IMRT planning. The full bladder, made feasible by the short treatment time with the LINACs, is intended to decrease the D50 to the bladder. An advantage of homogeneous CyberKnife SBRT and IMRT is that the nature of the plans limits severe hot spots which removes the need to identify the urethra as a critical structure.

The second dosimetric approach delivers a heterogeneous, or HDR-like, dose distribution (Fig. 3). As first described by Fuller et al. [27], using the CyberKnife, heterogeneous delivery uses over 200 beams to achieve a plan with postero-lateral hot spots that mimic HDR brachytherapy dosimetry. The rationale for this approach is based upon the belief that HDR brachytherapy's success not only may be due to hypofractionation but may also be a result of the higher dose delivered to the prostate periphery. Thus, by using heterogeneous planning and a dose that covers the PTV to 38 Gy in four fractions, the dosimetry of HDR 
Fig. 2 a Sagittal and $\mathbf{b}$ axial views of Trilogy treatment plan with rectal balloon. Image courtesy of Dr. R. Timmerman, University of Texas Southwestern

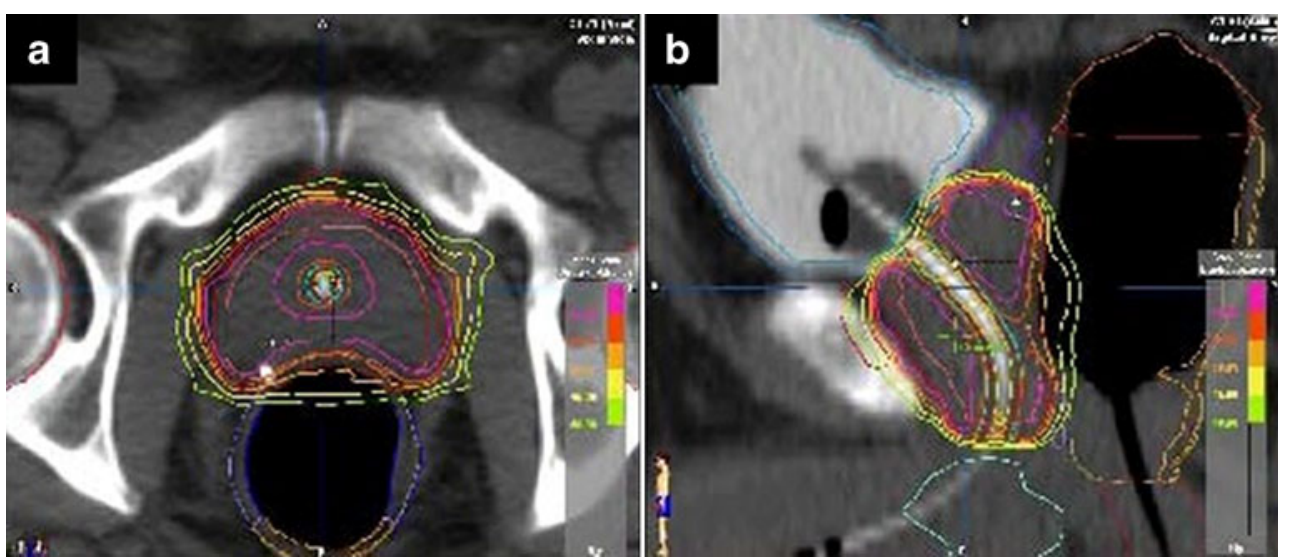

brachytherapy can be better emulated. Differences between the homogeneous and heterogeneous approaches include the PTV expansion beyond the prostate which is only $2 \mathrm{~mm}$ ( $0 \mathrm{~mm}$ posteriorly) and that the urethra is identified and subjected to critical structure dose constraints for heterogeneous plans. Using the heterogeneous approach, the V125 is $28-55 \%$ of the PTV, which approaches the V125s seen in HDR brachytherapy [27]. A potential advantage of this approach is that urethral doses can be kept even lower than used with HDR brachytherapy. To do this requires 230-318 beams which can take up to 90 min with older CyberKnife systems using two fixed collimators, as opposed to 4550 min with CyberKnife homogeneous plans [27]. Recent innovations have improved this scenario: the IRIS variable collimator (Accuray Inc.), for example, allows the use of multiple collimator sizes which are changed automatically throughout a treatment fraction. In addition, Sequential Optimization (Accuray Inc.) allows development of better,

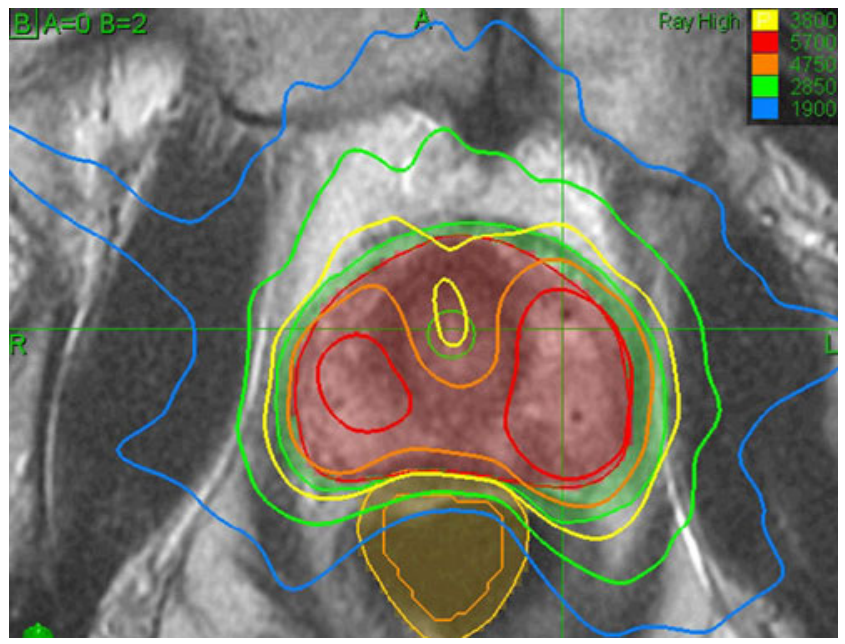

Fig. 3 Example heterogeneous, HDR-like CyberKnife treatment plan. The prescribed dose is 38 Gy delivered in 4 fractions. Notice the urethra sparing and very tight margins used with heterogeneous treatment. Image courtesy of Dr. D. Fuller, CyberKnife Centers of San Diego more efficient plans that can be delivered in a shorter time. Specifically, a recent update by Fuller shows that using the Iris variable collimator with sequential optimization can reduce the number of beams and monitor units and reduce the beam on time by $43 \%$ [35].

\section{Gantry-based SBRT clinical outcomes}

The first clinical outcomes of SBRT for prostate cancer came from Madsen et al. [36]. Using IMRT with six noncoplanar fields, 40 low-risk patients were treated to a total dose of 33.5 Gy delivered in five fractions. The dose was prescribed to the $D_{\max }$, and the entire prostate was covered by $90 \%$ of the prescribed dose. Treatment was well tolerated with only one late grade 3 urinary toxicity. However, the 4-year freedom from biochemical relapse rates were $70 \%$ and $90 \%$ using the ASTRO or Phoenix definitions, respectively. While the control rates are disappointing for SBRT, they are not surprising given that the $90 \%$ prescribed dose to the prostate is equivalent to a dose of 66 Gy delivered in 1.8 Gy fractions.

Since this initial SBRT result, only a few additional publications to date have reported on gantry based SBRT delivery (Table 2). In 2008, Tang et al. [37] reported on treatment of 30 patients with 35 Gy delivered in five fractions once a week over 29 days. They postulated that the hypothesized slow doubling time of prostate cancer should ameliorate any potential detrimental effect on tumor control with the weekly schedule. Preliminary results with 6-month follow-up reported no grade 3 toxicities; longer term results have not been published. More recently, Boike et al. [7] reported on a phase 1 SBRT study using Trilogy (Varian Medical Systems), Synergy (Elekta) or TomoTherapy (Accuray Inc.) for delivery. In this dose escalation study, three groups of 15 patients each received either 45, 47.5, or 50 Gy (the highest SBRT dose reported to date) delivered in five fractions QOD. A rectal balloon was used to push the posterior and lateral rectal walls away from the planning target volume (PTV) and to stabilize the prostate. Fiducial 
Table 2 Summary of published SBRT results to date with more than 12 months follow-up

\begin{tabular}{|c|c|c|c|c|c|c|c|}
\hline Study & Treatment & $\begin{array}{l}\text { No. of } \\
\text { patients }\end{array}$ & Risk group(s) & $\begin{array}{l}\text { Median follow-up } \\
\text { (months) }\end{array}$ & $\begin{array}{l}\text { Late grade } 3 \\
\text { GU toxicity }\end{array}$ & $\begin{array}{l}\text { Late grade } 3 \mathrm{GI} \\
\text { toxicity }\end{array}$ & FFBF \\
\hline \multicolumn{8}{|l|}{ Gantry-based systems } \\
\hline Madsen et al. [36] & 33.5 Gy in $5 \mathrm{fx}$ & 40 & Low & 41 & None & None & $\begin{array}{l}90 \% \text { 4-year } \\
\text { actuarial }\end{array}$ \\
\hline Boike et al. [7] & $45-50$ Gy in $5 \mathrm{fx}^{\mathrm{a}}$ & 45 & Low and int & $30,18,12$ & $4 \%$ & $\begin{array}{l}2 \% \text { plus } 1 \\
\text { grade } 4\end{array}$ & $100 \%$ \\
\hline Mantz et al. [11] & 40 Gy in $5 \mathrm{fx}^{\mathrm{a}}$ & 80 & Low & 36 & None & None & $100 \%$ \\
\hline \multicolumn{8}{|l|}{ Cyberknife } \\
\hline King et al. $[8,9]$ & $36.25 \mathrm{~Gy}$ in $5 \mathrm{fx}^{\mathrm{b}}$ & 67 & Low & 32 & $3.5 \%$ & None & $97 \%$ \\
\hline Friedland et al. [40] & 35 Gy in $5 \mathrm{fx}$ & 112 & Low, int, and high & 24 & $<1 \%$ & None & $98 \%$ \\
\hline Katz et al. $[10,41]$ & $35-36.25$ Gy in $5 \mathrm{fx}$ & 304 & Low, int and high & 48 & $2 \%$ & None & $\begin{array}{r}97 \%, 93 \%, 75 \% \\
4 \text {-year actuarial }\end{array}$ \\
\hline Freeman et al. [12] & $7-7.25$ Gy in $5 \mathrm{fx}$ & 41 & Low & 60 & 1 & None & $\begin{array}{c}93 \% 5 \text {-year } \\
\text { actuarial }\end{array}$ \\
\hline Bolzicco et al. [45] & 35 Gy in $5 \mathrm{fx}$ & 46 & Low and int & 20 & None & $2 \%$ & $100 \%$ \\
\hline Jabbari et al. [43] & $38 \mathrm{~Gy}$ in $4 \mathrm{fx}^{\mathrm{c}}$ & 38 & Low and int & 18 & $5 \%$ & None & $100 \%$ \\
\hline McBride et al. [13] & $36.25-37.5$ Gy in $5 \mathrm{fx}$ & 45 & Low & 44 & $<1 \%$ & None & $100 \%$ \\
\hline Fuller et al. [44] & $38 \mathrm{~Gy}$ in $4 \mathrm{fx}^{\mathrm{c}}$ & 54 & Low and int & 36 & $4 \%$ & None & $98 \%$ \\
\hline Kang et al. (39] & $32-36$ Gy in $4 \mathrm{fx}$ & 44 & Low, int, and high & 40 & None & None & $\begin{array}{l}100 \%, 100 \% \\
90.9 \%\end{array}$ \\
\hline
\end{tabular}

For clarity, when updated results are available, only the most recent publication is listed with earlier publications cited

$f x$ Fractions, int intermediate

${ }^{a}$ Delivered every other day

${ }^{\mathrm{b}}$ Delivered daily and/or every other day

${ }^{\mathrm{c}}$ Delivered with a heterogeneous dose distribution

markers and either megavoltage or kilovoltage clinical target (CT) were used for daily set-up, but intrafraction guidance was not used. A $3 \mathrm{~mm}$ expansion of the CT volume (CTV) was used to create the PTV. At a median followup of 30, 18 and 12 months for the three groups, no PSA failures were noted. The mean PSA was $0.2 \mathrm{ng} / \mathrm{ml}$ at 30 months. Overall, genitorinary (GU) grade 2 and grade 3 toxicity occurred in $31 \%$ and $4 \%$ of patients, respectively, and one grade $4 \mathrm{GU}$ toxicity occurred. Rectal grade 2 and grade 3 toxicity was found in $18 \%$ and $2 \%$ of patients, respectively.

Another approach to gantry-based SBRT delivery was reported at ASTRO 2011 by Mantz et al. [11] who used Trilogy with a Calypso system for daily patient set-up followed by cone beam CT to verify and check for deformation. Calypso was then further used to track intrafraction prostate motion. Eighty low-risk patients, none of which had a prostate larger than $60 \mathrm{cc}$, received 40 Gy delivered in five QOD fractions. At a median follow-up of 36.2 months, no biochemical failures were seen. The mean PSA at 3 years was $0.30 \mathrm{ng} / \mathrm{ml}$ down from $7.2 \mathrm{ng} / \mathrm{ml}$ before treatment. At 18 months, the mean EPIC scores for bowel, urinary and sexual function were lower than baseline but the changes were not significant changes from baseline.

\section{CyberKnife SBRT clinical outcomes}

While only a handful of clinical results exist on gantrybased SBRT for prostate cancer, there have been several publications for CyberKnife delivered SBRT reflecting the approximately 10,000 prostate patients treated with the CyberKnife since 2003. The apparent higher usage of CyberKnife SBRT for prostate cancer is likely due to its highly accurate real-time intrafraction image guidance that offers the precise delivery of the high dose-per-fraction treatment needed by SBRT as discussed earlier.

Among the early CyberKnife SBRT reports, researchers in Korea reported at ASTRO 2007 on delivery of a total of 32-36 Gy in four fractions to 44 patients [38] with minimal toxicity. Most of the patients were intermediate- or highrisk, likely explaining the 78\% 3-year actuarial freedom from biochemical failure rate. A recent update to this study [39], at a median 40-month follow-up, reports no failures have occurred in the intermediate-risk group and a $90 \%$ freedom from biochemical failure rate in the high-risk patients. Patients received androgen-suppression therapy for up to 2 years. No difference in biochemical control was seen with $32 \mathrm{~Gy}, 34 \mathrm{~Gy}$ or $36 \mathrm{~Gy}$, but the $36 \mathrm{~Gy}$ dose yielded higher urinary toxicity. 
The first prospective experience using CyberKnife was reported by King et al. from Stanford University [8]. In this phase 2 clinical trial, 41 low-risk hormone naïve patients received a dose of 36.25 Gy delivered in five fractions. Using only CT scans for treatment planning, the PTV was the prostate with a $5 \mathrm{~mm}$ margin, $3 \mathrm{~mm}$ posteriorly. The prescribed dose was normalized to the $89-90 \%$ isodose line, creating a highly homogeneous plan. At a median follow-up of 33 months, no failures were noted. The PSA response was robust; $78 \%$ of patients with a minimum of 12 -month follow-up achieved a PSA nadir of less $0.4 \mathrm{ng} / \mathrm{ml}$. Twentynine percent of patients had a benign PSA bounce. Late urinary toxicity rates included $24 \%$ grade 2 and $5 \%$ grade 3 with $15 \%$ late grade 2 rectal toxicity. Significant rectal side effects in the first 21 patients led to the decision to treat the latter 20 patients with a QOD schedule. Although the numbers are small, there appeared to be a decrease in side effects using the QOD schedule. For instance, 5 of 21 patients treated with daily fractions reported moderate to severe rectal problems, while 0 of 20 patients treated QOD reported such problems. Similarly, 4 of 21 patients treated daily reported severe late urinary problems, with two developing urethral stricture whereas only 1 of 20 patients treated QOD developed a significant late urinary toxicity. Although this difference was not statistically significant, these results led King et al. to recommend QOD treatment. A recent update reports on a total of 67 patients with two documented local failures from which they conclude that outcomes with SBRT is as least as good as conventional IMRT in terms of both toxicity and efficacy [9].

Numerous retrospective studies have been published (Table 2). Friedland et al., from Naples, FL reported on 112 patients (102 low-, 9 intermediate-, and 1 high-risk) treated with CyberKnife SBRT to a total dose of 35 Gy delivered in 5 daily fractions [40]. Treatment planning was similar to that used by King et al. with the same margins and the objective to deliver at least $95 \%$ of the prescribed dose to the PTV. An additional difference was that MRI was obtained and fused with the CT scan images to facilitate contouring and planning. At a median 24-month follow-up, the freedom from biochemical failure rate was $98 \%$. Two patients had local failures and one additional patient failed distantly with bone metastases. Almost all patients with more than 2 years follow-up had a PSA of less than $1.0 \mathrm{ng} / \mathrm{ml}$. AUA scores tended to revert to pretreatment levels after 3 months; one patient had a grade 3 GU toxicity requiring a TURP and one had rectal bleeding (grade 2). Potency was retained in $82 \%$ of patients at 2 years. This study was updated at ASCO 2010 by Freeman et al. [12]; at 30 months median follow-up local control was $97 \%$ for 152 patients and $81 \%$ of patients retained potency.

Katz et al. [10] published the largest CyberKnife SBRT series to date with treatment of 304 prostate cancer patients.
A small minority of patients received hormone ablative therapy that was discontinued prior to treatment. The first 50 patients, most of whom were low risk, received a total dose of 35 Gy delivered in 5 fractions. Homogeneous treatment plans were created on CT fused with MRI, using 5-mm margins to the PTV, $3 \mathrm{~mm}$ posteriorly. A typical plan covered $95 \%$ of the PTV to the $83-87 \%$ isodose line. The mean D50 to the rectum and bladder was $42 \%$, mean penile bulb dose was 18 Gy and mean testicular dose was 5 Gy. Two collimators were used to deliver $140-170$ beams. All patients received $1500 \mathrm{mg}$ of Amifostine (MedImmune, LLC Gaithersburg, MD) intrarectally 15 min prior to each fraction. At a median 30-month follow-up, there were no biochemical failures and the median PSA was $0.22 \mathrm{ng} / \mathrm{ml}$ with $97 \%$ of patients obtaining a PSA below $1.0 \mathrm{ng} / \mathrm{ml}$. Toxicity was mild with no grade 3 toxicity and only $2 \%$ of patients exhibiting late grade 2 urinary toxicity. The subsequent 254 patients (166 low-, 76 intermediate-, and 12 highrisk patients) received daily doses of 7.25 Gy delivered in 5 daily fractions. Treatment plans were similar to the first 50 patients except that the GTV included the proximal seminal vesicles if the patient was intermediate or high risk. At a median 17-month follow-up, there was one local failure in the high-risk group and two distant failures for both the lowand high-risk groups. A PSA bounce was observed in 19\% of patients. Urinary toxicity was slightly higher than in the 35 Gy group. Overall potency preservation was $87 \%$ at 2 years and the mean EPIC sexual score dropped by $20 \%$. The authors concluded that patients reported as good, if not better, QOL scores as with other forms of radiation therapy. In a recent update at ASTRO 2011, Katz et al. [41] reported $97 \%, 93 \%$, and $75 \%$ 4-year actuarial freedom from biochemical failure rates in the low-, intermediate- and highrisk patients, respectively. At an overall median follow-up of 48 months the median PSA was $0.2,0.1$, and $0.1 \mathrm{ng} / \mathrm{ml}$ at 36,48 , and 60 months, respectively. There was more urinary toxicity in the higher dose group with $10 \%$ late grade 2 and $2 \%$ late grade 3 toxicity (differences were not statistically significant). Potency was retained in $78 \%$ of patients.

The first results with a median 5-year follow-up were published by Freeman and King [42] who pooled 41 low-risk patients who received 35-36.25 Gy delivered in five fractions. Late GU toxicity consisted of $25 \%$ grade $1,7 \%$ grade 2 , and $2.5 \%$ grade 3 . Late GI toxicity was $13.5 \%$ for grade 1 and $2.5 \%$ for grade 2 . The 5-year actuarial freedom from biochemical failure was $93 \%$. Of the three patients, two who failed received the lower dose of $35 \mathrm{~Gy}$, whereas the other received $36.25 \mathrm{~Gy}$.

In support of these results, several other recent CyberKnife SBRT studies report on treatment of 50 or fewer patients. Jabbari et al. treated 20 low-risk patients with a total dose of 38 Gy delivered in four fractions using an HDR-like dose distribution [43]. An excellent PSA response was seen at a median of 18-month follow-up with 2 grade 3 
urinary toxicities. At ASTRO 2011, Fuller et al. [44] reported on 49 low- and intermediate-risk patients treated with 38 Gy delivered in four fractions using an HDR-like dose distribution with 2-mm margins everywhere except posteriorly where no margin was used. Acute urinary toxicity was mild. At a median follow-up of 36 months, late GU toxicity consisted of $18 \%$ grade 2 and $4 \%$ grade 3 . Late rectal toxicity was extremely low, presumably due to the very tight posterior margins. The median PSA was $0.4,0.2$, and $0.1 \mathrm{ng} / \mathrm{ml}$ at 24 , 36 , and 48 months, respectively. The 3 -year actuarial freedom from biochemical failure rate was $96 \%$. Bolzicco et al. [45] treated 46 low- and low-intermediate risk patients with a total dose of 35 Gy delivered in five fractions. At a median followup of 20 months, no biochemical failures occurred and low toxicity was observed. Both Aluwini et al. [46] and Townsend et al. [47] published feasibility studies showing mild and transient early toxicity following delivery of $38 \mathrm{~Gy}$ in four fractions or 35-37.5 Gy in five fractions, respectively. Kang et al. reported excellent results on 44 intermediate- and high-risk patients receiving 32-36 Gy in four fractions in combination with up to 2 years of hormone ablative therapy. At a median 40-month follow-up, the intermediate-risk patients had no failures, and the high-risk patients had a $90 \%$ freedom from biochemical failure rate. A multi-institutional phase 1 trial reported on delivery of 36.25-37.5 Gy in five fractions for 45 low-risk patients [13]. At a median follow-up of 44 months, no biochemical failures occurred with toxicities consisting of one grade 3 urinary and two grade 3 rectal late toxicities.

The above results consistently show low recurrence rates and very low PSA nadirs, which appear to be better than results reported with delivery of $78-81 \mathrm{~Gy}$. If the $\alpha / \beta$ ratio were $3 \mathrm{~Gy}$, then delivery of $35 \mathrm{~Gy}$ in five fractions would yield an EQD1.8 of $70 \mathrm{~Gy}$, which would not be expected to outperform the results achieved with $81 \mathrm{~Gy}$. Consequently, the very low reported recurrence rates at 4-5 years appear to only be possible with an $\alpha / \beta$ ratio of 1.5 Gy or less. Similarly, the observed PSA responses support an $\alpha / \beta$ ratio of 1.5 Gy or less. For instance, Katz et al. report a 4-year median PSA of $0.1 \mathrm{ng} / \mathrm{ml}$ [41]; in comparison, Jabbari et al. [48] reported a 5year median PSA of $0.4 \mathrm{ng} / \mathrm{ml}$ with delivery of 79.2 Gy 3DCRT. Not only does this indicate a more robust response to SBRT, but it also suggests a highly durable one, as recent studies show a correlation of low PSAs with excellent longterm outcomes [49-51].

Further examining the safety and efficacy of CyberKnifedelivered SBRT, two Accuray-sponsored CyberKnife studies have completed accrual and will soon add another few hundred patients to the literature. In one of the studies, $40 \mathrm{~Gy}$ is delivered in 5 days in a homogeneous distribution [52], and the other $38 \mathrm{~Gy}$ is delivered in 4 days using heterogeneous or HDR-like dosimetry [53]. Early results on these studies were presented at ASTRO 2010 showing low toxicity and promising PSA responses [54, 55].

\section{SBRT as a boost for higher risk disease}

For intermediate- and high-risk disease, there is a significant risk of extraprostatic involvement [56], yet the localized nature of HDR or low-dose rate (LDR) implants limits their ability to adequately treat microscopic disease outside the capsule. Consequently, researchers have used brachytherapy as a boost within the prostate following EBRT or IMRT delivery of 45-50 Gy to the prostate and surrounding tissues. As noted earlier, HDR brachytherapy as a boost to EBRT or IMRT can achieve 5-8 year freedom from biochemical failure rates of $85-90 \%$ and $68-73 \%$ for intermediate- and high-risk disease, respectively $[23,57]$. This boost strategy has been employed in three published SBRT studies all of which have used the CyberKnife for the SBRT boost. Jabbari et al. [43] treated 18 patients with intermediate- and high-risk disease with an SBRT boost of 19.5 Gy delivered in two fractions after 45-50 Gy IMRT pelvic radiation therapy. In addition to the boost these patients received hormone ablative therapy for up to 2 years. At 23.5-month median follow-up, no biochemical failures had occurred, and there was no acute grade 3 toxicity. Two patients had late grade $3 \mathrm{GU}$ toxicity. Oermann et al. reported on early results following treatment of 24 intermediate- and high-risk patients with an SBRT boost of 19.5 Gy delivered in three fractions following 50.4 Gy IMRT. At 6-month follow-up, the median PSA dropped to $1.5 \mathrm{ng} / \mathrm{ml}$ and the EPIC scores returned to baseline. Katz et al. [58] treated 73 patients ( 41 intermediate- and 32 high-risk patients) with an SBRT boost of 18-21 Gy in three fractions 2 weeks after delivery of four-field EBRT to 45 Gy in 25 fractions. Amifostine was used as a rectal radioprotection prior to the CyberKnife boost and 36 patients received ADT for a mean 4.8 months prior to SBRT. At a median 33-month follow-up, the freedom from biochemical failure rate was $89.5 \%$ and $78 \%$ for the intermediate- and high-risk patients, respectively. Two high-risk and no intermediate-risk patients failed locally. A PSA nadir of less than $0.5 \mathrm{ng} / \mathrm{ml}$ was obtained for $71.8 \%$ of patients. Toxicity was mild with no late grade 3 rectal and 1 grade 3 late urinary complications. The three boost dose schemes of $18 \mathrm{~Gy}, 19.5$ Gy and 21 Gy yielded no difference in efficacy or toxicity and there was no difference in outcomes with or without hormone ablation. These outcomes are similar to those reported with HDR brachytherapy as a boost at the same follow-up interval and the reported EPIC QOL measures appear as good if not better than those reported by Sanda et al. [59] for LDR brachytherapy boost. A recent update (Katz et al. submitted) at a median 57-month follow-up shows no additional toxicity and a 5 -year actuarial freedom from biochemical failure of $87.5 \%$ and $73 \%$ for intermediate- and high-risk patients. These results suggest the efficacy of SBRT boost will continue to be equivalent to HDR brachytherapy, however, additional follow-up is needed to confirm this. 
Is a boost necessary for more aggressive tumors?

There are two rationales for using SBRT as a boost following EBRT or IMRT for intermediateand high-risk patients rather than using SBRT monotherapy: extraprostatic extension and pelvic node coverage. The potentially limited coverage of extraprostatic extension by HDR and LDR brachytherapy resulted in the use of these treatments as a boost for intermediate- and high-risk diseases. In the case of SBRT, however, the immediate extracapsular regions and the seminal vesicles can be adequately covered by the PTV expansion. Thus, it appears unnecessary to use EBRT prior to SBRT for the purpose of covering extracapsular extension. At ESTRO 2010, Katz et al. compared 44 intermediate-risk patients treated with CyberKnife SBRT alone (total dose 36.25 Gy with a 5-mm margin) to 35 intermediate-risk patients treated with 45 Gy minipelvis followed by a CyberKnife SBRT boost (total dose 18-21 Gy). None of these patients received ADT. At a median 41-month follow-up, no statistically significant difference in the freedom from biochemical failure rates $(94.3 \%$ for boost vs. $95.5 \%$ for SBRT alone) was observed. In addition, the 36-month median PSA levels were identical $(0.2 \mathrm{ng} / \mathrm{ml}$ in both groups). This suggests that delivering a high BED to the prostate with a sufficient dose to the margin may maximize control, regardless of whether SBRT is used alone or in conjunction with EBRT. This is further supported by Katz et al. who observed a 4-year freedom from biochemical failure rate of $93 \%$ with a median PSA of $0.1 \mathrm{ng} / \mathrm{ml}$ for 81 intermediate-risk patients, most of whom received a dose of 36.25 Gy in five fractions [41].

In the case of high-risk patients, pelvic node coverage is the primary rationale for using boost rather than monotherapy. Two randomized trials have examined the use of whole pelvic XRT to treat the lymph nodes $[60,61]$. Both of these studies failed to show a benefit in treating the lymph nodes to prophylactic doses for high-risk patients. Further questioning the need for pelvic node coverage, excellent 5 -year freedom from biochemical failure rates of $68 \%$ and $70 \%$ have been obtained for high-risk patients using both conventional [62] and moderate hypofractionation [63] without pelvic radiation, respectively. Furthermore, in an editorial, Nguyen et al. [64] state that the lack of evidence supporting whole pelvic treatment suggests it should not be offered routinely in the clinic. This raises the question as to whether such treatment should be recommended for use in conjunction with SBRT for higher-risk patients. Published SBRT outcomes for high-risk patients suggest pelvic treatment is not necessary. Kang et al. [39] recently reported on 29 highrisk patients treated with CyberKnife SBRT alone to a dose of 34-36 Gy in four fractions in conjunction with ADT. At a median 40-month follow-up, the 5-year freedom from biochemical failure rate was $90.8 \%$. In addition, Katz et al. observed a 4-year freedom from biochemical failure rate of
$75 \%$ for 12 patients treated with SBRT alone. The observed control rates in these studies suggest that outcomes for high risk disease may be as good, with less toxicity, as with SBRT as a boost to pelvic radiotherapy. However, given these are retrospective studies with limited numbers of patients receiving varying amounts of ADT, additional data is needed to verify this observation.

\section{SBRT for recurrent disease}

An additional application of SBRT for prostate cancer is the treatment of recurrent disease using SBRT either in the prostate or in the lymph nodes. The limited data in the literature regarding re-irradiation of such patients focuses on HDR brachytherapy [65-67]. Nevertheless, it would seem imperative for patients whose prostates are being re-irradiated to severely limit the urethral dose. SBRT delivered through the CyberKnife or through LINAC-based IMRT treatment plans offer the advantage of such urethral dose constraints. Jereczek-Fossa et al. [68] reported on 34 patients treated with CyberKnife SBRT for recurrences. Sixteen patients with solitary lymph node recurrence received 33 Gy in three fractions [68]; 15 patients with local recurrences following EBRT received 30 Gy in five fractions; and 4 patients with anastomotic recurrence following surgery received $30 \mathrm{~Gy}$ in five fractions. For those patients whose whole gland was treated following EBRT failure, the urethra dose was limited to $125 \%$ of the prescription dose. At a median 16.9-months follow-up, 5 of the 16 lymph node recurrence patients; 5 of 15 EBRT failure patients, and 2 of 4 anastomotic recurrence patients had biochemical progression. Two grade 3 acute urinary and two grade 3 late urinary toxicities were observed to date. In addition, Fuller [69] has delivered CyberKnife SBRT to a dose of 34 Gy delivered in five fractions for 5 patients with local failure following EBRT. At a maximum follow-up of 18 months, one patient was lost to follow-up and four patients had a significant drop in PSA similar to that seen with SBRT monotherapy treatment. Toxicity was mild. We must await results with larger numbers of patients and longer follow-up to assess the safety and efficacy of this approach. Toward this end, the CyberKnife Centers of San Diego are currently accruing patients in a phase 2 study examining the use of CyberKnife SBRT for patients with locally recurrent disease (NCT00851916).

\section{Considerations of SBRT for prostate cancer}

Approaches to reduce rectal toxicity

While the rectal toxicity observed with prostate SBRT has been mostly mild and moderate, caution has been employed 
in some protocols to limit any potential rectal toxicity including uses of a radioprotectant or a rectal balloon. Katz et al. [10] used Amifostine as a rectally administered radioprotectant prior to delivery of each SBRT fraction. Amifostine was used because it has resulted in a significant decrease in RTOG grade 2 toxicity when administered prior to EBRT [70]. In the Katz et al. study using Amiofostine, mild rectal toxicity was observed but the rectal toxicity rate does not appear to be substantially different than toxicity reported by others $[8,9$, $40,42]$ delivering the same doses of 35-36.6 Gy in five fractions. Amifostine use can be mildly toxic (such as grade 1 rash) for a small number of patients when used intrarectally and it has a significant cost. Thus, given no substantial clinical advantage, there does not appear to be a need to use Amifostine routinely prior to SBRT. In addition, this author has observed when treating with the CyberKnife, the use of the IRIS collimator can decrease the D50 to the rectum substantially, which reduces rectal toxicity risk.

In 2002, Teh et al. [71] reported on use of a rectal balloon while delivering hypofractionated IMRT with the NOMOS Peacock system and the MIMiC treatment delivery system (Sewickley, PA). They concluded that with the rectal balloon there was significant reduction of prostate motion and a reduction in the total rectal volume receiving a high dose. Sanghani et al. showed that use of a rectal balloon in 3D conformal delivery reduced the median percent of rectal volume receiving 70 Gy from $25 \%$ to $3.6 \%$ which they postulated could decrease the rate of late rectal bleeding [72]. Rectal wall sparing through the use of a rectal balloon coupled with the potential for decreased rectal toxicity was further corroborated by van Lin et al. [73]. Boike et al. [7] used a rectal balloon in their phase 1 dose escalation study in which the balloon appears to significantly improve the DVHs to the lateral and posterior rectal walls. Figure 2 shows an example treatment plan with a rectal balloon. As can be seen the plan exhibits high conformality with a tight isodose curve around that prostate and a low dose to the rectum. The use of a rectal balloon appears to be helpful in the absence of intrafractional tracking, as the prostate position is stabilized. When intrafractional tracking is performed, as with the CyberKnife, rectal balloon use is unnecessary.

\section{Testicular dose}

With noncoplanar delivery using the CyberKnife several beams can traverse the testicles if the treatment plans are not specifically created to avoid such traversal. King cautioned that this can produce a dose of up to 6.6 Gy to the testicles [74]. King further postulated that since a testicular dose of 2-4 Gy can produce hypogonadism, it is possible that the excellent PSA declines seen with SBRT may at least partially be attributed to declining testosterone levels. Fuller [75] responded by noting neither a significant decline in measured serum testosterone levels nor a decline in sexual desire was observed after SBRT in his patients. Thus, he concluded that the median PSA of $0.4 \mathrm{ng} / \mathrm{ml}$ at 2 years for his cohort was entirely due to radiation effect. Both King and Fuller agreed that an effort should, nevertheless, be made to reduce dose to the testicles by removing beams that traverse through them. King demonstrated that this can bring the testicular dose down to 1.2 Gy without having a detrimental effect on the quality of the plan. Katz et al. [10] measured the dose to the testicles for 12 patients and found a mean dose in the $5 \mathrm{~Gy}$ range. While testosterone levels were not specifically measured, no clinical symptoms of hormone ablation were observed in any of the 304 patients treated. Oermann et al. [76] recently published a study of 26 patients treated with CyberKnife SBRT to a total dose of $36.25 \mathrm{~Gy}$ in five fractions. They reported a median baseline serum testosterone level of $13.81 \mathrm{nmol} / \mathrm{L}$ with a clinically insignificant decline to a median level of $10.53 \mathrm{nmol} / \mathrm{L}$ at 1 year. EPIC QOL sexual functions showed a clinically insignificant decline that returned to baseline by 1 year after treatment. Consequently, the authors concluded that this slight drop in testosterone level did not contribute to the large drop in median PSA observed at 1 year follow-up. Testicular dose has also been examined for IMRT where as much as 5 Gy can be delivered to the testicles [77], yet clinically significant declines in testosterone have not been reported. Thus, while it remains important to limit testicular dose through careful treatment planning, SBRT-triggered hypogonadism does not appear to be the cause of the excellent PSA declines observed following treatment.

\section{Erectile dysfunction}

Only a few publications have reported erectile dysfunction rates following SBRT. Of note, Friedland et al. report an $82 \%$ potency preservation rate 1 year following SBRT delivery of 35 Gy [40]; Katz et al. [41] report $80 \%$ potency preservation 40 months following SBRT deliver of 35-36.25 Gy; and Wiegner et al. [78] report baseline erectile dysfunction of $38 \%$ increased to $71 \%$ ( $40 \%$ potency preservation rate) at a median 35.5 months following SBRT delivery of $36.25 \mathrm{~Gy}$. A variety of differences in these studies could account for the $80-82 \%$ vs $40 \%$ observed potency rates following SBRT. The higher dose of Wiegner et al. could be seen as a factor for the lower potency preservation rate in comparison to Friedland et al. Yet, Katz et al. saw no difference in potency rates between dose groups of 35 and $36.25 \mathrm{~Gy}$. Another important difference between these studies lies in the treatment details. In particular, Katz et al. and Friedland et al. fused MRI scans into the planning CT scans, whereas Wiegner et al. used only CT scans. MRI scans provide a more detailed view of the prostate, especially at the apex. For example, Sannazzari et al. [79] found that the use of MRIs in treatment planning decreased 
prostate volumes by $34 \%$. Perhaps when not using MRIs smaller GTVs are used which result in lower doses to the neurovascular bundles and subsequently higher potency rates; an analysis of the GTV contours used by the various studies is necessary to confirm this conjecture. Further follow-up with large numbers of patients may also be helpful in addressing this question.

\section{Hormonal ablation therapy}

Hormonal therapy has often been recommended for intermediate- to high-risk patients with EBRT, based on results reported by Bolla et al. and D'Amico et al $[80,81]$. These studies showed benefit from using hormones for EBRT doses up to $70 \mathrm{~Gy}$. Martinez et al. indicated no benefit to hormone ablation using HDR brachytherapy hypofractionation with delivery of high BEDs. One explanation for this disparity could be that any advantage provided by hormonal ablation therapy diminishes as the BED to the prostate increases. Supporting this, Katz et al. also found no benefit to hormonal ablative therapy for intermediate- and high-risk SBRT boost patients. The duration of hormonal ablative therapy is also likely a factor in that a longer duration of hormonal therapy may extend the time to observed biochemical failure. For example, Kang et al. [39] observed $100 \%$ and $90 \%$ 40-month freedom from biochemical failure rates for intermediate- and high-risk patients, respectively, for patients who received hormonal ablation therapy for up to 2 years following SBRT. These very high rates of freedom from biochemical failure suggest longer follow-up on these patients is needed to determine the effects of the long-term hormonal therapy. Nevertheless, based on the favorable monotherapy results for intermediate-risk patients and the toxicity of hormone ablation, few CyberKnife treatments incorporate the use of hormonal ablative therapy suggesting SBRT has an advantage over standard fractionation as the side effects of hormone ablation may be avoidable for intermediate-risk patients.

What is the optimal dose?

The published studies to date have employed a wide range of doses extending from $33.75 \mathrm{~Gy}$ in five fractions to $50 \mathrm{~Gy}$ in five fractions. In addition, there is variation in the interval between fractions, with King et al.and Boike et al. employing QOD schedules. Ultimately, the goal of any radiation treatment is to use the minimal dose necessary to control the cancer while limiting side effects. This is especially important in the treatment of men with low-risk disease who stand a very low chance of dying of their disease within 10 years if left untreated. The absence of large, prospective SBRT studies with long-term follow-up makes selecting an optimal dose difficult. Nevertheless, given its importance it seems pertinent to explore the currently available data in hope of identifying some trends.
For instance, the 5-year freedom from biochemical failure rate for low-risk disease is around $90 \%$ with delivery of 81 Gy at 1.8 Gy per fraction [21]. Delivery of 86.4 Gy at 1.8 Gy per fraction increased the 5 -year freedom from biochemical failure rate to $95 \%$ [63]. In contrast, delivery of 70 Gy in 28 fractions [15] and delivery of $66 \mathrm{~Gy}$ in 22 fractions [16] achieved 5-year freedom from biochemical failure rates of $95 \%$ and $98 \%$, respectively. These comparable control rates with various fractionation schemes can be explained by a prostate cancer $\alpha / \beta$ of 1.5 Gy (Table 1). Given an $\alpha / \beta$ ratio of $1.5 \mathrm{~Gy}, 66$ Gy delivered in 22 fractions is equivalent to $90 \mathrm{~Gy}$, which coupled with the very high control rate for 66 Gy suggests a dose response from 81 to 90 Gy EQD. Since many SBRT dose schemes use EQD1.8 $\mathrm{s}$ much greater than $90 \mathrm{~Gy}$, the question arises as to whether there continues to be a greater response above $90 \mathrm{~Gy}$.

A new study by Katz et al. [82] explores the question of optimal dose for lower risk patients through a matched pair analysis of two groups of 41 patients with low or low- intermediate risk. One group received $35 \mathrm{~Gy}$ in five daily fractions (EQD1.8 approximately $91 \mathrm{~Gy}$ ) and one group received 36.25 Gy (EQD1.8 approximately 96 Gy) also in five daily fractions. At a median 48-month follow-up, each group had only one failure yielding a freedom from biochemical failure rate of $97.5 \%$. At 36 and 48 months, the median PSAs were identical between the dose groups at 0.2 and $0.1 \mathrm{ng} / \mathrm{ml}$. In the higher dose group, late grade 2 urinary toxicity was slightly higher than the lower dose group and there was one grade 3 toxicity in the higher dose group but none in the lower dose group. These differences were not statistically significant, probably due to the small numbers. Despite the small numbers and limited follow-up, these data raise that question as to whether 35 Gy in five fractions (EQD1.890 Gy) is the optimal dose to control lower risk disease with minimal side effects. It is interesting to compare the PSA results achieved by heterogeneous planning to a dose of $38 \mathrm{~Gy}$ in four fractions to $35 \mathrm{~Gy}$ in five fractions given homogeneously. At ASTRO 2011, Fuller et al. [44], using heterogeneous and Katz et al. [41], using homogeneous planning, both reported the exact same median PSA in populations of low- and intermediate-risk patients. Specifically, both reported median PSAs of 0.4, 0.2 , and $0.1 \mathrm{ng} / \mathrm{ml}$ at 24,36 , and 48 months, respectively. One implication from this observation is that the ultimate radiobiological effect from the two doses may be the same which, if confirmed with longer follow-up and more patients, would further support the concept that 35 Gy in five fractions may be the threshold dose to maximize tumor kill.

Lastly, Kang et al. [39] reported $100 \%$ and $90 \%$ 40-month freedom from biochemical failure rates for 44 intermediateand high-risk patients who received 32-36 Gy in four fractions. No difference in control was seen with 32,34 , or $36 \mathrm{~Gy}$, but the 36 Gy dose yielded higher urinary toxicity. While they now use 32-34 Gy it is important to note that these patients also received up to 2 years of hormonal therapy. Thus, while 
they see sufficient control, even for more aggressive disease, longer follow-up is needed to differentiate the impact of the hormonal therapy. Overall, the small number of patients and short follow-up of these studies prohibit firm conclusions. Nevertheless, caution is warranted as these studies suggest higher doses, with their threat of higher toxicity, may not be necessary. Perhaps when more data is available from the two Accuray sponsored clinical studies, more conclusions can be drawn.

\section{Future considerations}

As more published outcomes become available supporting SBRT for prostate, cancer researchers have began exploring alternative and enhanced SBRT treatment. One such enhancement may be to include functional imaging information in treatment planning. Assessment of hypervascularity using dynamic contrast-enhanced MRI [83] or 3D power Doppler ultrasound [84] offers the potential for integrating biologically defined focal boosts into SBRT. For example, high-risk patients may be treated with 35 Gy to the whole prostate in combination with an integrated boost of 38 40 Gy to identified areas of high-risk. Such an approach has the advantage of ensuring a limit to the urethral dose while providing a targeted boost.

Another area of exploration is the possibility of even further decreasing the number of fractions. Martinez et al. has explored this possibility, decreasing the number of delivered HDR brachytherapy fractions to even as low as one fraction [85]. Muacevic et al. have a pilot study treating 17 low-risk patients with CyberKnife delivery of one 19 Gy fraction including delivery of 28 Gy posterolaterally. At a mean 16-month follow-up, the treatment was well tolerated (personal communication, A. Muacevic, CyberKnife Center Munich Grosshadern), but it is early to compare efficacy with the more standard four to five fraction schemes.

\section{Summary}

The increase in the therapeutic ratio offered by SBRT combined with the convenient, rapid treatment schedule that lasts only 4-5 days and the potentially lower cost provided by outpatient treatment has motivated clinicians to increasingly apply SBRT to prostate cancer over the last several years. The number of published studies on SBRT for prostate cancer continues to grow with follow-up now extending to 5 years. Thus far, the results have demonstrated excellent control rates, low PSA nadirs, long time to PSA nadir and overall low morbidity suggesting a bright future for SBRT. As the number of men in their 60 s and above increases within the overall population health care resources will become strained. The shorter treatment times and lower cost of SBRT is likely to become a useful tool in controlling costs while preserving good outcomes. Ultimately, randomized trials comparing SBRT to other forms of radiation, and even surgery, are needed to confirm the superiority of this approach. If performed, however, such studies will take several years. In the interim, the excellent efficacy and limited toxicity observed thus far combined with a short, noninvasive treatment that has a minimal impact on the patients' life argues for acceptance of SBRT as a viable option available for prostate cancer patients today.

Acknowledgments I gratefully acknowledge the editorial assistance of Pam Commike, PhD of Accuray Inc.

\section{References}

1. Brenner DJ, Martinez AA, Edmundson GK et al (2002) Direct evidence that prostate tumors show high sensitivity to fractionation (low alpha/beta ratio), similar to late-responding normal tissue. Int J Radiat Oncol Biol Phys 52:6-13

2. Fowler JF (2005) The radiobiology of prostate cancer including new aspects of fractionated radiotherapy. Acta Oncol 44:265-276

3. Miralbell R, Roberts SA, Zubizarreta E et al (2012) DoseFractionation Sensitivity of Prostate Cancer Deduced from Radiotherapy Outcomes of 5,969 Patients in Seven International Institutional Datasets: alpha/beta $=1.4(0.9-2.2)$ Gy. Int J Radiat Oncol Biol Phys 82(1):17-24

4. Brenner DJ (2004) Fractionation and late rectal toxicity. Int J Radiat Oncol Biol Phys 60:1013-1015

5. Fowler J, Chappell R, Ritter M (2001) Is alpha/beta for prostate tumors really low? Int J Radiat Oncol Biol Phys 50:1021-1031

6. Shaffer R, Pickles T, Lee R et al (2011) Deriving prostate alphabeta ratio using carefully matched groups, long follow-up and the phoenix definition of biochemical failure. Int J Radiat Oncol Biol Phys 79:1029-1036

7. Boike TP, Lotan Y, Cho LC et al (2011) Phase I dose-escalation study of stereotactic body radiation therapy for low- and intermediate-risk prostate cancer. J Clin Oncol 29:2020-2026

8. King CR, Brooks JD, Gill H et al (2009) Stereotactic body radiotherapy for localized prostate cancer: interim results of a prospective phase II Clinical Trial. Int J Radiat Oncol Biol Phys 73:10431048

9. King CR, Brooks JD, Gill $\mathrm{H}$ et al Long-term outcomes from a prospective trial of stereotactic body radiotherapy for low-risk prostate cancer. Int J Radiat Oncol Biol Phys 2011

10. Katz AJ, Santoro M, Ashley R et al Stereotactic body radiotherapy for organ-confined prostate cancer. BMC Urol 2010;10:1

11. Mantz C, Fernandez E, Zucker I et al (2011) A phase II trial of realtime target tracking SBRT for low-risk prostate cancer utilizing the calypso 4D localization system: patient-reported quality of life and toxicity outcomes. Int J Radiat Oncol Biol Phys 81:S100

12. Freeman DE, Friedland JL, Masterson-McGary ME (2010) Stereotactic radiosurgery for low-intermediate risk prostate cancer: an emerging treatment approach. J Clin Oncol 33:208

13. McBride SM, Wong DS, Dombrowski JJ et al Hypofractionated stereotactic body radiotherapy in low-risk prostate adenocarcinoma: preliminary results of a multi-institutional phase 1 feasibility trial. Cancer 2011 
14. Lukka HR. A randomized phase II trial of hypofractionated radiotherapy for favorable risk prostate cancer-RTOG CCOP Study; 2011.RTOG Website

15. Kupelian PA, Willoughby TR, Reddy CA et al (2007) Hypofractionated intensity-modulated radiotherapy (70 Gy at 2.5 Gy per fraction) for localized prostate cancer: Cleveland clinic experience. Int J Radiat Oncol Biol Phys 68:1424-1430

16. Rene N, Faria S, Cury F et al (2010) Hypofractionated radiotherapy for favorable risk prostate cancer. Int J Radiat Oncol Biol Phys $77: 805-810$

17. Arcangeli G, Saracino B, Gomellini S et al (2010) A prospective phase III randomized trial of hypofractionation versus conventional fractionation in patients with high-risk prostate cancer. Int $\mathbf{J}$ Radiat Oncol Biol Phys 78:11-18

18. Pollack A, Walker G, Buyyounouski M et al (2011) Five Year results of a randomized external beam radiotherapy hypofractionation trial for prostate cancer. Int J Radiat Oncol Biol Phys 81:S1

19. Ritter MA, Forman JD, Kupelian PA et al (2011) Five-year efficacy and toxicity outcomes from a phase $\mathrm{i} / \mathrm{ii}$ trial of increasingly hypofractionated radiation therapy for prostate cancer. Int J Radiat Oncol Biol Phys 81:S99

20. Kupelian PA, Thakkar VV, Khuntia D et al (2005) Hypofractionated intensity-modulated radiotherapy (70 gy at 2.5 Gy per fraction) for localized prostate cancer: long-term outcomes. Int J Radiat Oncol Biol Phys 63:1463-1468

21. Zelefsky MJ, Chan H, Hunt M et al (2006) Long-term outcome of high dose intensity modulated radiation therapy for patients with clinically localized prostate cancer. J Urol 176:1415-1419

22. Collins CD, Lloyd-Davies RW, Swan AV (1991) Radical external beam radiotherapy for localised carcinoma of the prostate using a hypofractionation technique. Clin Oncol (R Coll Radiol) 3:127132

23. Demanes DJ, Rodriguez RR, Schour L et al (2005) High-dose-rate intensity-modulated brachytherapy with external beam radiotherapy for prostate cancer: California endocurietherapy's 10-year results. Int J Radiat Oncol Biol Phys 61:1306-1316

24. Martinez AA, Demanes J, Vargas C (2010) High-dose-rate prostate brachytherapy: an excellent accelerated-hypofractionated treatment for favorable prostate cancer. Am J Clin Oncol 33:481-488

25. Demanes DJ, Martinez AA, Ghilezan M (2011) High-dose-rate monotherapy: safe and effective brachytherapy for patients with localized prostate cancer. Int J Radiat Oncol Biol Phys 81:1286-1292

26. Yoshioka Y, Konishi K, Sumida I et al (2011) Monotherapeutic high-dose-rate brachytherapy for prostate cancer: five-year results of an extreme hypofractionation regimen with 54 Gy in nine fractions. Int J Radiat Oncol Biol Phys 80:469-475

27. Fuller DB, Naitoh J, Lee C et al (2008) Virtual HDR CyberKnife treatment for localized prostatic carcinoma: dosimetry comparison with HDR brachytherapy and preliminary clinical observations. Int J Radiat Oncol Biol Phys 70:1588-1597

28. Balter JM, Sandler HM, Lam K et al (1995) Measurement of prostate movement over the course of routine radiotherapy using implanted markers. Int J Radiat Oncol Biol Phys 31:113-118

29. Kron T, Thomas J, Fox C et al (2010) Intra-fraction prostate displacement in radiotherapy estimated from pre- and post-treatment imaging of patients with implanted fiducial markers. Radiother Oncol 95:191-197

30. King CR, Lehmann J, Adler JR et al (2003) CyberKnife radiotherapy for localized prostate cancer: rationale and technical feasibility. Technol Cancer Res Treat 2:25-30

31. Hossain S, Xia P, Chuang C et al (2008) Simulated real time image guided intrafraction tracking-delivery for hypofractionated prostate IMRT. Med Phys 35:4041-4048

32. Dawood O, Sims S, Dooley J et al (2009) Impact of robotic IMRT on normal tissue sparing in the treatment of prostate cancer as compared to conventional IMRT. J Urol 183:674-675
33. Xie Y, Djajaputra D, King CR et al (2008) Intrafractional motion of the prostate during hypofractionated radiotherapy. Int J Radiat Oncol Biol Phys 72:236-246

34. Lei S, Piel N, Oermann E et al (2010) Six-dimensional correction of intra-fractional prostate motion with CyberKnife stereotactic body radiation therapy. Front Radiat Oncol 1:48

35. Fuller DB, Naitoh J, Reilly M et al (2009) Virtual HDR prostate cyberknife radiosurgery: efficacy, toxicity and quality of life. 2009 CyberKnife Users' Meeting, Hollywood

36. Madsen BL, Hsi RA, Pham HT et al (2007) Stereotactic hypofractionated accurate radiotherapy of the prostate (SHARP), 33.5 Gy in five fractions for localized disease: first clinical trial results. Int $\mathrm{J}$ Radiat Oncol Biol Phys 67:1099-1105

37. Tang CI, Loblaw DA, Cheung P et al (2008) Phase I/II study of a five-fraction hypofractionated accelerated radiotherapy treatment for low-risk localised prostate cancer: early results of pHART3. Clin Oncol (R Coll Radiol) 20:729-737

38. Choi C, Cho G, Kim K et al (2007) Stereotactic radiation therapy of localized prostate cancer using CyberKnife. Int J Radiat Oncol Biol Phys 69:S375

39. Kang JK, Cho CK, Choi CW et al (2011) Image-guided stereotactic body radiation therapy for localized prostate cancer. Tumori 97:43-48

40. Friedland JL, Freeman DE, Masterson-McGary ME et al (2009) Stereotactic body radiotherapy: an emerging treatment approach for localized prostate cancer. Technol Cancer Res Treat 8:387-392

41. Katz AJ, Santoro M, DiBlasio F et al (2011) Stereotactic body radiation therapy for low, intermediate, and high-risk prostate cancer: disease control and quality of life. Int J Radiat Oncol Biol Phys 81:S100

42. Freeman DE, King CR (2011) Stereotactic body radiotherapy for low-risk prostate cancer: five-year outcomes. Radiat Oncol 6:3

43. Jabbari S, Weinberg VK, Kaprealian T et al Stereotactic body radiotherapy as monotherapy or post-external beam radiotherapy boost for prostate cancer: technique, early toxicity, and psa response. Int $\mathrm{J}$ Radiat Oncol Biol Phys 2012

44. Fuller DB, Shirazi R, Naitoh J et al (2011) Virtual HDR SBRT For Localized prostatic carcinoma: efficacy and quality of life assessment. Int J Radiat Oncol Biol Phys 81:S423-S424

45. Bolzicco G, Favretto MS, Scremin E et al (2010) Image-guided stereotactic body radiation therapy for clinically localized prostate cancer: preliminary clinical results. Technol Cancer Res Treat 9:473-477

46. Aluwini S, van Rooij P, Hoogeman M et al (2010) CyberKnife stereotactic radiotherapy as monotherapy for low- to intermediatestage prostate cancer: early experience, feasibility, and tolerance. $\mathrm{J}$ Endourol 24:865-869

47. Townsend NC, Huth BJ, Ding W et al (2011) Acute toxicity after cyberknife-delivered hypofractionated radiotherapy for treatment of prostate cancer. Am J Clin Oncol 34:6-10

48. Jabbari S, Weinberg VK, Shinohara K et al (2010) Equivalent biochemical control and improved prostate-specific antigen nadir after permanent prostate seed implant brachytherapy versus highdose three-dimensional conformal radiotherapy and high-dose conformal proton beam radiotherapy boost. Int J Radiat Oncol Biol Phys 76:36-42

49. Ray ME, Thames HD, Levy LB et al (2006) PSA nadir predicts biochemical and distant failures after external beam radiotherapy for prostate cancer: a multi-institutional analysis. Int J Radiat Oncol Biol Phys 64:1140-1150

50. Zelefsky MJ, Kuban DA, Levy LB et al (2007) Multi-institutional analysis of long-term outcome for stages T1-T2 prostate cancer treated with permanent seed implantation. Int J Radiat Oncol Biol Phys 67:327-333

51. Stock RG, Klein TJ, Cesaretti JA et al (2009) Prognostic significance of 5-year PSA value for predicting prostate cancer recurrence after 
brachytherapy alone and combined with hormonal therapy and/or external beam radiotherapy. Int J Radiat Oncol Biol Phys 74:753-758

52. Meier R 2008 CyberKnife Radiosurgery for organ-confined prostate cancer: homogenous dose distribution.

53. Fuller DB 2008CyberKnife radiosurgery for low \& intermediate risk prostate cancer: emulating HDR brachytherapy dosimetry

54. Fuller DB, Mardirossian G, Wong D et al (2010) Prospective Evaluation of stereotactic radiotherapy for low and intermediate risk prostate cancer: emulating HDR brachytherapy dose distribution. Int J Radiat Oncol Biol Phys 78:S358-S359

55. Meier R, Beckman A, Kaplan I et al (2010) Stereotactic radiotherapy for organ-confined prostate cancer: early toxicity and quality of life outcomes from a multi-institutional trial. Int J Radiat Oncol Biol Phys 78:S57

56. Partin AW, Yoo J, Carter HB et al (1993) The use of prostate specific antigen, clinical stage and Gleason score to predict pathological stage in men with localized prostate cancer. J Urol 150:110-114

57. Galalae RM, Kovacs G, Schultze J et al (2002) Long-term outcome after elective irradiation of the pelvic lymphatics and local dose escalation using high-dose-rate brachytherapy for locally advanced prostate cancer. Int J Radiat Oncol Biol Phys 52:81-90

58. Katz A, Santoro M, Ashley R et al (2010) Stereotactic body radiotherapy as boost for organ-confined prostate cancer. Technol Cancer Res Treat 9:575-582

59. Sanda MG, Dunn RL, Michalski J et al (2008) Quality of life and satisfaction with outcome among prostate-cancer survivors. N Engl J Med 358:1250-1261

60. Lawton CA, DeSilvio M, Lee WR et al (2007) Results of a phase II trial of transrectal ultrasound-guided permanent radioactive implantation of the prostate for definitive management of localized adenocarcinoma of the prostate (radiation therapy oncology group 98-05). Int J Radiat Oncol Biol Phys 67:39-47

61. Pommier P, Chabaud S, Lagrange JL et al (2007) Is there a role for pelvic irradiation in localized prostate adenocarcinoma? Preliminary results of GETUG-01. J Clin Oncol 25:5366-5373

62. Kupelian P, Willoughby T, Mahadevan A et al (2007) Multiinstitutional clinical experience with the Calypso System in localization and continuous, real-time monitoring of the prostate gland during external radiotherapy. Int J Radiat Oncol Biol Phys 67:1088-1098

63. Cahlon O, Zelefsky MJ, Shippy A et al (2008) Ultra-high dose (86.4 Gy) IMRT for localized prostate cancer: toxicity and biochemical outcomes. Int J Radiat Oncol Biol Phys 71:330-337

64. Nguyen PL, D'Amico AV (2008) Targeting pelvic lymph nodes in men with intermediate- and high-risk prostate cancer despite two negative randomized trials. J Clin Oncol 26:2055-2056, author reply 2056-2057

65. Beyer DC (2004) Salvage brachytherapy after external-beam irradiation for prostate cancer. Oncology (Williston Park) 18:151-158, discussion 158-160, 163-154

66. Grado GL, Collins JM, Kriegshauser JS et al (1999) Salvage brachytherapy for localized prostate cancer after radiotherapy failure. Urology 53:2-10

67. Lee B, Shinohara K, Weinberg V et al (2007) Feasibility of highdose-rate brachytherapy salvage for local prostate cancer recurrence after radiotherapy: the University of California-San Francisco experience. Int J Radiat Oncol Biol Phys 67:1106-1112

68. Jereczek-Fossa BA, Beltramo G, Fariselli L et al Robotic Imageguided stereotactic radiotherapy, for isolated recurrent primary, lymph node or metastatic prostate cancer. Int J Radiat Oncol Biol Phys 2011
69. Fuller DB, Virtual HDR (2011) CyberKnife treatment for localized prostatic carcionoma: principles and clinical update. In: Ponsky LE (ed) Treating prostate cancer and related genitourinary applications. Springer, Berline, pp 155-163

70. Simone NL, Menard C, Soule BP et al (2008) Intrarectal amifostine during external beam radiation therapy for prostate cancer produces significant improvements in quality of life measured by EPIC score. Int J Radiat Oncol Biol Phys 70:90-95

71. Teh BS, McGary JE, Dong L et al (2002) The use of rectal balloon during the delivery of intensity modulated radiotherapy (IMRT) for prostate cancer: more than just a prostate gland immobilization device? Cancer J 8:476-483

72. Sanghani MV, Ching J, Schultz D et al (2004) Impact on rectal dose from the use of a prostate immobilization and rectal localization device for patients receiving dose escalated 3D conformal radiation therapy. Urol Oncol 22:165-168

73. van Lin EN, Hoffmann AL, van Kollenburg P et al (2005) Rectal wall sparing effect of three different endorectal balloons in $3 \mathrm{D}$ conformal and IMRT prostate radiotherapy. Int J Radiat Oncol Biol Phys 63:565-576

74. King CR, Lo A, Kapp DS (2009) Testicular dose from prostate cyberknife: a cautionary note. Int J Radiat Oncol Biol Phys 73:636-637, author reply 637

75. Fuller DB (2009) Testicular dose from prostate cyberknife: a cautionary note in regard to King et al. Int $\mathrm{J}$ Radiat Oncol Biol Phys 73:637

76. Oermann EK, Suy S, Hanscom HN et al (2011) Low incidence of new biochemical and clinical hypogonadism following hypofractionated stereotactic body radiation therapy (SBRT) monotherapy for low- to intermediate-risk prostate cancer. J Hematol Oncol 4:12

77. Yogeswaren S, Teh B, Mai W et al (2004) Radiation dose to testicles and serum testosterone levels in low risk prostate cancer patients undergoing intensity-modulated radiation therapy (IMRT). Int J Radiat Oncol Biol Phys 60:S456

78. Wiegner EA, King CR (2010) Sexual Function after stereotactic body radiotherapy for prostate cancer: results of a prospective clinical trial. Int J Radiat Oncol Biol Phys 78:442-448

79. Sannazzari GL, Ragona R, Ruo Redda MG et al (2002) CT-MRI image fusion for delineation of volumes in three-dimensional conformal radiation therapy in the treatment of localized prostate cancer. Br J Radiol 75:603-607

80. Bolla M, Van Tienhoven G, Warde P et al (2010) External irradiation with or without long-term androgen suppression for prostate cancer with high metastatic risk: 10-year results of an EORTC randomised study. Lancet Oncol 11:1066-1073

81. D'Amico AV, Chen MH, Renshaw AA et al (2008) Androgen suppression and radiation vs radiation alone for prostate cancer: a randomized trial. JAMA 299:289-295

82. Katz AJ, Santoro M, Ashley R et al (2011) Stereotactic body radiation therapy for low- and low-intermediate-risk prostate cancer: is there a dose effect? Front Radiat Oncol 1:49

83. Low RN, Fuller DB, Muradyan N (2011) Dynamic gadoliniumenhanced perfusion MRI of prostate cancer: assessment of response to hypofractionated robotic stereotactic body radiation therapy. AJR Am J Roentgenol 197:907-915

84. Sauvain JL, Palascak P, Bourscheid D et al (2003) Value of power doppler and $3 \mathrm{D}$ vascular sonography as a method for diagnosis and staging of prostate cancer. Eur Urol 44:21-30, discussion 30-21

85. Bea umont launches study of single-dose radiation treatment for prostate cancer; 2011 